\title{
Accurate Monte Carlo critical exponents for Ising lattices
}

\author{
Jorge García and Julio A. Gonzalof \\ Departamento de Física de Materiales, \\ Universidad Autónoma de Madrid \\ Cantoblanco, 28049 Madrid, Spain. \\ Manuel I. Marqués用 \\ Center for Polymer Studies and Department of Physics, Boston University \\ Boston MA 02215, USA.
}

(Dated: October 26, 2018)

\begin{abstract}
A careful Monte Carlo investigation of the phase transition very close to the critical point $(T \longrightarrow$ $\left.T_{c}, H \longrightarrow 0\right)$ in relatively large $\mathrm{d}=3, \mathrm{~s}=1 / 2$ Ising lattices did produce critical exponents $\beta_{3 D}$ $=0.3126(4) \cong 5 / 16, \delta_{3 D}^{-1}=0.1997(4) \cong 1 / 5$ and $\gamma_{3 D}=1.253(4) \cong 5 / 4$. Our results indicate that, within experimental error, they are given by simple fractions corresponding to the linear interpolations between the respective two-dimensional (Onsager) and four-dimensional (mean field) critical exponents. An analysis of our inverse susceptibility data $\chi^{-1}(T)$ vs. $\left|T-T_{c}\right|$ shows that these data lead to a value of $\gamma_{3 D}$ compatible with $\gamma^{\prime}=\gamma$ and $T_{c}=4.51152(12)$, while $\gamma$ values obtained recently by high and low temperature series expansions and renormalization group methods are not.
\end{abstract}

PACS numbers: 64.60.-i, 64.60.Fr, 64.60.Cn.

\section{INTRODUCTION}

The Ising model[1], rightly considered as the prototype of statistical systems with non-classic power law critical behavior, has been extensively investigated for many years. Systems with short-range interactions display Ising-like critical behavior, f.i. liquid-vapor, multicomponent fluid mixtures, uniaxial magnets, etc, and there is a wealth of very accurate experimental information on these systems.

To describe f.i. the behavior of a $s=1 / 2$ uniaxial ferromagnet near the critical point $\left(T=T_{c}, H=0\right)$ two critical exponents, $\beta$ for the spontaneous magnetization $M_{s}(T)$ and $\delta^{-1}$ for the field dependence of the magnetization $M_{c}(H)$ at the critical temperature, determine basically the critical behavior through $M_{s}(T) \sim\left|T_{c}-T\right|^{\beta}$ and $M_{c}(H) \sim H^{1 / \delta}$. It is well known[2] that for a two dimensional Ising lattice $(d=2)$ Onsager's solution gives fractional values for $\beta_{2 D}=1 / 8$ and $\delta_{2 D}^{-1}=1 / 15$. For a four dimensional Ising lattice $(d=4)$ on the other hand, the critical exponents are the mean field exponents[3], given also by fractional values, $\beta_{4 D}=1 / 2$ and $\delta_{4 D}^{-1}=1 / 3$.

It is a legitimate question to ask whether for a threedimensional Ising lattice $(d=3)$, for which no general theoretical solution is available for the moment, the values for $\beta_{3 D}$ and $\delta_{3 D}^{-1}$ are rational fractions or not. In fact, almost forty years ago, Cyril Domb, one of the very pioneers in the then rapidly growing field of phase transitions, suggested that for three-dimensional Ising lattices the susceptibility critical exponent $\gamma=\beta(\delta-1)$ might

\footnotetext{
*Electronic address: ig.garcia@uam.es

$\dagger$ Electronic address: ulio.gonzalo@uam.es

$\ddagger$ Electronic address: manuel@argento.bu.edu
}

be given by the fractional value $\gamma_{3 D}=5 / 4=1.25$. Since then a tremendous amount of work (experimental, theoretical and computational) has been performed with the aim to get ever more precise numerical characterizations of the phase transitions. Table I gives a representative sample [4] of numerical values 5 , 6, 7, 8, 9, 10, 11, 12] for the exponents $\gamma_{3 D}$ and $\beta_{3 D}$ obtained by various methods: high temperature expansion series, low temperature expansion series, Monte Carlo simulations and field theoretical methods. The overall picture of the numerical values for $\gamma_{3 D}$ and $\beta_{3 D}$ is reasonably good, and they seem to favor $\gamma_{3 D}<1.25$ and $\beta_{3 D}>0.3125$, but, clearly, the uncertainties quoted in parentesis cannot be taken strictly at face value.

In the present work we present results on critical exponents values based upon optimized accurate Monte Carlo calculations and we investigate to what extent the $d=3$ Ising exponents are compatible with the simple fractions interpolated between the fractional $d=2$ Ising exponents and the, fractional too, $d=4$ Ising exponents. In particular we will use direct determinations of $\beta_{3 D}$, from $M_{s}(T)$ data at $H=0$, and of $\delta_{3 D}^{-1}$, from $M_{c}(H)$ data at $T=T_{c}$, as well as $\chi^{-1}(T)$ vs $T$, including data both below $T_{c}$ (LT phase) and above $T_{c}$ (HT phase), which allow us to make internal consistency checks, so that the actual value used for the critical temperature can be confirmed to be compatible with the scaling requirement $\gamma^{\prime}=\gamma$ or not.

In order to establish the reliability of the data and the propriety of the method of analysis used we will proceed in two steps. First we will check data on large twodimensional lattices, for which the fractional values of the exponents are known exactly, and then we will analyze data on large three-dimensional lattices for which the fractional values proposed are only educated guesses. Our data, therefore, can either lend support or leave un- 


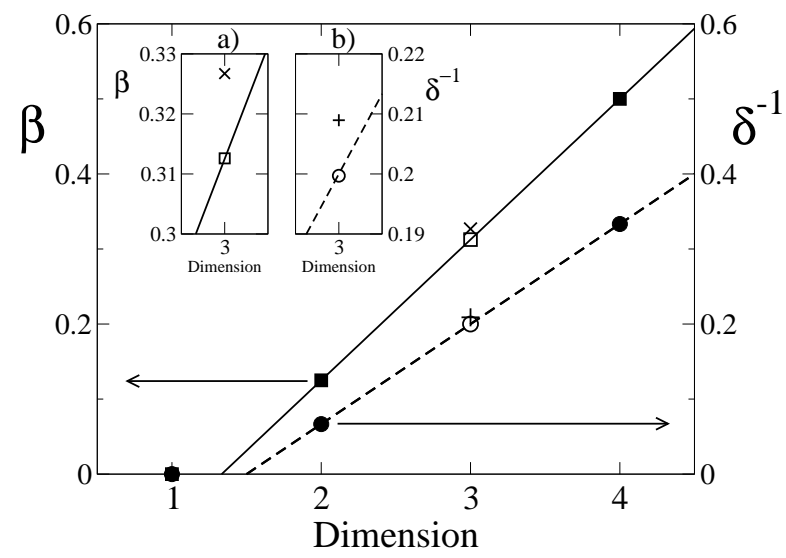

FIG. 1: $\beta_{3 D}$ and $\delta_{3 D}^{-1}$ (open symbols) obtained by interpolation between exact two-dimensional data from Onsager [2] and four-dimensional data from mean field 21] theory (filled symbols). The crosses show current accepted results [0 In all cases, error bars are smaller than the symbol's size. Insets a) and b) are a blow-up close to the $d=3$ region for $\beta$ and $\delta^{-1}$, respectively.

supported the fractional values proposed. Figure 1 shows the evolution of critical exponents with lattice dimensionality $d=2,3,4, \ldots$ for Ising systems.

\section{MONTE CARLO DETERMINATIONS OF $M_{s}(T)$ AND $M_{c}(T)$}

Finite size scaling Monte Carlo simulations of phase transitions are known to be among the best and more effective techniques available to characterize the critical behavior of model systems such as Ising systems of any dimensionality. To determine critical exponents with the highest possible reliability it is desirable (i) to increase systematically the size $L$ of the system till the results become practically independent of $L$ within error bars; (ii) to make the interval (temperature or magnetic field) between successive states in the vicinity of the critical point as small as possible, choosing wisely the full range (temperature of magnetic field) so that is not too large (time consuming) or too small (inconvenient of incomplete) to determine adequately the exponent in question; (iii) to insure that the time spent in the calculation at each point (temperature, magnetic field) is sufficient to arrive to true equilibrium. We did use two-dimensional lattices of $800^{2}, 900^{2}$ and $1000^{2}$ spins, and three-dimensional lattices of $90^{3}, 100^{3}$ and $115^{3}$ spins. The temperature intervals were of the order of 0.001 , in units of $T$ such that $T_{c 2 D}=2.269185314213 \ldots$ and $T_{c 3 D}=4.51152(12)$ for determinations of the spontaneous magnetization $M_{s}(T)$, and field intervals of the order of 0.0005 for determinations of $M_{c}(H)$ at the critical isotherm.

The number of Monte Carlo steps taken to insure equilibrium at each state was 50000 and the number of states considered in the partition function was 10000 . No improvement was detected by increasing the number of states. To get $M_{s}(T)$ a Wolff[14 algorithm was used, which is a generalization of the Swendsen-Wang 115$]$ algorithm. To obtain $M_{c}(H)$, a somewhat novel but straightforward, calculation not usually found in the literature, a standard Metropolis 16 algorithm was used and it was proved to be quite adequate.

Initially both periodic boundary conditions and free boundary conditions were used. After confirming that for very large lattices the difference between results obtained with either set of boundary conditions was quite negligible, we used subsequently periodic boundary conditions all the way.

For the temperature scans we always began at $T>T_{c}$ and spent sufficient time at the beginning to make sure that thermal equilibrium was attained already far above the transition temperature. Nevertheless the statistical ups and downs in the residual $M(T)$ data at $T>T_{c}$, are much larger than the corresponding fluctuations at $T<T_{c}$ which become almost negligible and display a beautiful continuity all the way down in temperature. The log-log plots of $M$ vs. $\left|T-T_{c}\right|$ and $M$ vs. $H$ indicate clearly, first that we have been able to get really close to the critical point, more so than in other Monte Carlo calculations for which data in the literature are sufficiently explicit, and, second, that in the full range displayed for $T$ or $H$ corrections to scaling are invisible, which make the data especially apt to determine the exponents $\beta_{3 D}$ and $\delta_{3 D}^{-1}$.

\section{MONTE CARLO RESULTS FOR TWO-DIMENSIONAL LATTICES}

Figure 2(a) gives a log-log plot of the spontaneous magnetization as a function of temperature for a squared Ising lattice with $1000 \times 1000$ spins. Finite size effects show up as rounding at $\left|T-T_{c}\right| \longrightarrow 0$ which occurs only at $\left|T-T_{c}\right| \lesssim 0.002$ with $T_{c}=2.269185314213 \ldots$ Corrections to scaling should appear at the other end of the temperature range examined, but are quite invisible in our data. The spontaneous magnetization data give directly a value for $\beta_{2 D}=0.1242 \pm 0.0008 \cong 1 / 8$, as expected. Figure 2(b) presents a log-log plot of the magnetization as a function of field for the same squared Ising lattice with $1000 \times 1000$ spins at the critical isotherm. Finite size effects begin to appear as incipient rounding at $H \lesssim 0.0001$ but are almost imperceptible. Corrections to scaling should appear at the other end of the field range investigated but are completely negligible in our data. These critical isotherm data, not previously investigated in depth, as far as we know, give directly $\delta_{2 D}^{-1}=0.06656$ $\pm 0.00022 \cong 1 / 15$, as expected.

These results lend support to the expectation that carefully taken Monte Carlo data in large enough lattices taken at small enough temperature / field intervals are accurate enough to investigate whether critical expo- 
TABLE I: Some estimated $s=1 / 2$ critical exponents for $d=3$ Ising simple cubic lattices

\begin{tabular}{|c|c|c|c|c|}
\hline Method & Reference & Year & $\gamma$ & $\beta$ \\
\hline \multirow{2}{*}{ High $T$ expansions } & [5] & $(2002)$ & $1.2368(10)$ & $0.3243(30)^{a}$ \\
\hline & 河 & (1997) & $1.2388(10)$ & $0.3278(13)^{a}$ \\
\hline \multirow[t]{2}{*}{ Low $T$ expansions } & (7) & (1993) & $1.251(28)$ & $0.329(9)$ \\
\hline & [ & (1991) & $1.255(10)$ & $0.320(3)$ \\
\hline \multirow[t]{2}{*}{ Monte Carlo } & ]9] & $(1999)$ & $1.2372(13)^{b}$ & $0.3269(5)$ \\
\hline & 10 & $(2000)$ & $1.255(18)^{b}$ & $0.325(5)$ \\
\hline \multirow[t]{2}{*}{ Field Theoretical } & 11 & $(2001)$ & $1.2403(8)$ & $0.3257(5)$ \\
\hline & 12 & (1994) & 1.258 & 0.336 \\
\hline
\end{tabular}

${ }^{a}$ Obtained using the scaling relation $\beta=\nu(1+\eta) / 2$

${ }^{b}$ Obtained using the scaling relation $\gamma=(2-\eta) / \nu$

nents are given by simple fractions or not, at least in the case of two dimensions.

A straightforward numerical analysis of the fractions compatible with the experimental results and the uncertainties quoted has been made. For $d=2$ it can be seen that $n / m$ with $n<m$ compatible with the uncertainties, must go to $m$ values very large, $m>256$ for $\beta_{2 D}$ and $m>360$ for $\delta_{2 D}^{-1}$.

\section{MONTE CARLO RESULTS FOR THREE-DIMENSIONAL LATTICES}

Figure 3(a) gives a log-log plot of $M_{s}(T)$ vs. $T$ for a simple cubic lattice with $115 \times 115 \times 115$ spins. Lattices with $90 \times 90 \times 90$ and $100 \times 100 \times 100$ spins were investigated and the results, of course slightly less accurate, where completely consistent with those obtained with the larger lattice. Again finite size effects appear at $\left|T-T_{c}\right| \longrightarrow 0$, and they appear clearly at $\left|T-T_{c}\right| \lesssim 0.004$ but corrections to scaling are invisible in the temperature range investigated. For $\beta_{3 D}$ we get

$$
\beta_{3 D}=0.3126(4) \cong \frac{5}{16}
$$

Figure 3(b) produces a similar log-log plot of the magnetization as a function of field for the same lattice with $115 \times 115 \times 115$ spins at $T=T_{c}=4.51152(12)$. It is clear that both finite size effects and systematic departures from scaling are absent or imperceptible in the field range explored. These new, not previously directly investigated critical isotherm data, result in

$$
\delta_{3 D}^{-1}=0.1997(4) \cong \frac{1}{5}
$$

which differs somewhat from previous indirect estimates of $\delta_{3 D}$ summarized by A. Pelissetto and E. Vicari [4] as giving $\delta_{3 D}=4.789(2)$, equivalent to $\delta_{3 D}^{-1}=$ $0.2088(1)$.
Using the numerical values for $\beta_{3 D}$ and $\delta_{3 D}^{-1}$ in Equations (11) and (2) one gets $\gamma_{3 D}$ indirectly as

$$
\gamma_{3 D}=\beta_{3 D}\left(\delta_{3 D}-1\right)=1.252(4)
$$
$\delta$.

by means of the scaling relation for $\gamma$ in terms of $\beta$ and

But one can get $\gamma$ directly from plots of susceptibility data at $T>T_{c}(\gamma)$ as well as at $T<T_{c}\left(\gamma^{\prime}\right)$ giving us the opportunity to check that the fundamental equality $\gamma=\gamma^{\prime}$, confirmed by renormalization group theory [4], is fulfilled.

Figure 3(c) gives log-log plots of direct Monte Carlo data on the susceptibility $\chi^{-1}(T)$ as a function of $\mid T-$ $T_{c} \mid$ both above and below the critical temperature $T_{c}$ $=4.51152$. The resulting directly determined value of gamma is

$$
\gamma_{3 D}=\gamma_{3 D}^{\prime}=1.253(4)
$$

which is in excellent agreement with the forty-year-old prediction of Domb and Sykes 17.

As mentioned before the following consistency check was made: changing smoothly the critical temperature value by increments (decrements) of 0.00002 we can obtain smooth changes in the effective values of the exponents $\gamma_{3 D}$ and $\gamma_{3 D}^{\prime}$ to reproduce numerical values as low as 1.237 for $\gamma_{3 D}$, resulting in $\gamma_{3 D}^{\prime}>1.25$, or as high as 1.263 for $\gamma_{3 D}$, resulting in $\gamma_{3 D}^{\prime}<1.25$. With our Monte Carlo data, which in sufficiently wide ranges appear to be free of finite size effects as well as of corrections to scaling effects, only using the right $T_{c}=4.51152$, in perfect agreement with the values quoted in the literature, the requirement $\gamma=\gamma^{\prime}$ is duly fulfilled.

Table II shows that according to our data for simple cubic $s=1 / 2$ Ising lattices, simple fractions for $\beta$ and $\delta^{-1}$ (the same procedure could be employed to check simple fractions for $\gamma$ ) compatible with the quoted uncertainties are given always by $n / m$ fractions which are, either identical to the interpolated values $\beta_{3 D}=5 / 16, \delta_{3 D}^{-1}=1 / 5$ $\left(\gamma_{3 D}=5 / 4\right)$ or much more complex fractions involving $m>128$ (for $\beta$ ) and $m>285$ (for $\delta^{-1}$ ). 
TABLE II: Monte Carlo $s=1 / 2$ Ising critical exponents in a three-dimensional simple cubic lattice of $115 \times 115 \times 115$ spins

\begin{tabular}{lc}
\hline \hline$\beta$ & $n / m^{c}$ \\
$0.31254 \pm 0.00029$ & $5 / 16, \ldots, 40 / 128(41 / 131)$ \\
\hline$\delta^{-1}$ & $n / m^{c}$ \\
$0.1997 \pm 0.0004$ & $1 / 5, \ldots, 57 / 285(57 / 286)$ \\
\hline \hline
\end{tabular}

${ }^{c}$ Fractional values compatible with the quoted uncertaintities up to $m_{\max }=128$ for $\beta$ and up to $m_{\max }=285$ for $\delta^{-1}$. More complex fractions (shown in parenthesis) are compatible with the uncertaintities only with denominators larger than 128 and 285, respectively.

\section{CONCLUDING REMARKS}

Our Monte Carlo data do not prove directly beyond doubt that three-dimensional Ising lattices are characterized by the fractional critical exponents $\beta_{3 D}=5 / 16$ and $\delta_{3 D}^{-1}=1 / 5$ (and through the corresponding scaling relations, by the resulting critical exponents $\gamma=5 / 4$, $\alpha=1 / 8, \nu=5 / 8$ and $\eta=0$ ) but they support strongly and consistently this fractional values, in particular the susceptibility data resulting in $\gamma_{3 D}=\gamma_{3 D}^{\prime}=$ $1.25=5 / 4$. Our empirical data may be the basis for future well grounded theoretical arguments confirming the above fractional critical exponents.

\section{Acknowledgments}

We acknowledge helpful comments by Lidia Braunstein and Gerry Paul to Jorge García and Manuel I. Marqués during fruitful stays at the Boston University Physics Department. Support from the Spanish Ministry of Science and Technology through Grant Number BFM2000-0032 is gratefully acknowledged. M.I. Marqués acknowledges the Postdoctoral Grant at Boston University from the Spanish Ministry of Education.
[1] See f.i. J. Adler, J. Phys. A 16, 3585 (1983).

[2] L. Onsager, Phys. Rev 65, 117 (1944).

[3] J.M. Yeomans, Statistical Mechanics of Phase Transitions, (Oxford University Press, 1992).

[4] See f.i. A. Pelissetto and E. Vicari, Phys. Rep 368, 549727 (2002).

[5] P. Butera and M. Comi, Phys. Rev. B 65, 144431 (2002).

[6] P. Butera and M. Comi, Phys. Rev. B 56, 8212 (1997).

[7] A.J. Guttmann and I.G. Enting, J. Phys. A 26, 806 (1993).

[8] J. Oitman, C.J. Hamer and W. Zheng, J. Phys. A 24, 2863 (1991).

[9] H.W.J. Blöte, L.N. Shchur and A.L. Talapov, Int. J. Mod. Phys. C 10, 137 (1999).

[10] N. Ito, K. Hukushima, K. Ogawa and Y. Ozeki, J. Phys. Soc. Japan 69, 1931 (2000).

[11] F. Jasch and H. Kleinert, J. Math. Phys. 42, 52 (2001).

[12] J. Berges, N. Tetradis and C. Wetterich, Phys. Rev. Lett. 77, 873 (1996).
[13] S. Caracciolo, G. Ferraro and A. Pelissetto, J. Phys. A 24, 3625 (1991).

[14] U. Wolff, Phys. Rev. Lett. 62, 361 (1989).

[15] R.H. Swendsen and J.S. Wang, Phys. Rev. Lett.. 58, 86 (1987).

[16] N. Metropolis, A.W. Rosenbluth, M.N. Rosenbluth, A.H. Teller and E. Teller, J. Chem. Phys. 21, 1087 (1953).

[17] Domb and Sykes, Phys. Rev. 128, 168-173 (1962).

[18] C. Domb, in Phase Transitions and Critical Phenomena, edited by C. Domb and M.S. Green (Academic, London, 1974).

[19] C. Domb, The Critical Point (Taylor\&Francis, London, 1996).

[20] M.E. Fisher, Rev. Mod. Phys. 46, 597 (1962); 70, 653 (1998).

[21] See f.i. J.A. Gonzalo, Effective Field Approach to Phase Transitions and some Applications to Ferroelectrics (World Scientific, Singapore, 1991). 

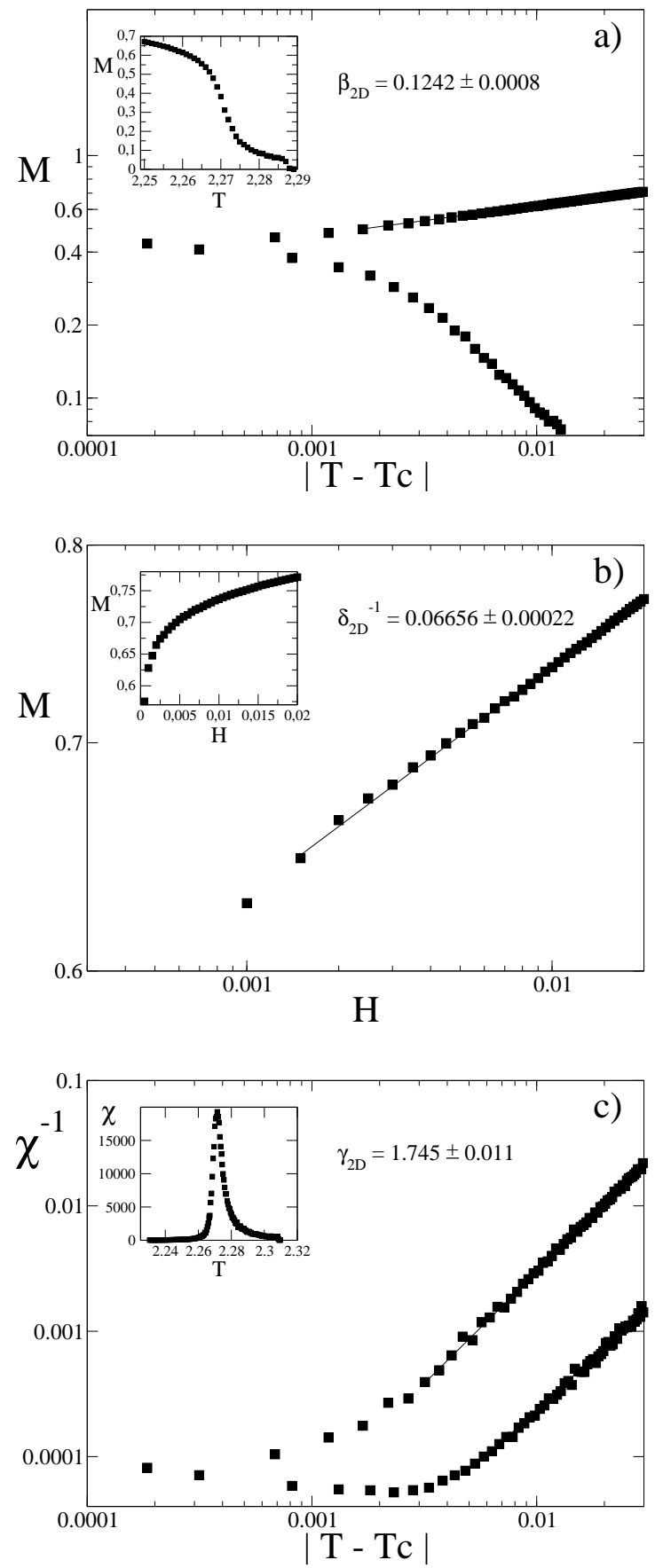

FIG. 2: Two dimensional critical exponents $\beta_{2 D}, \delta_{2 D}^{-1}$ and $\gamma_{2 D}$ obtained by fits of Monte Carlo data of spontaneous magnetizacion $M_{s}(H=0)$ as a function of temperature $T$ near $T_{c}=2.269185314213$, critical isotherm, $M$ as a function of $H$ at $T=T_{c}$, and isothermal susceptibility $\chi$, respectively, in a system of $1000 \times 1000$ spins. Insets show the row data. 

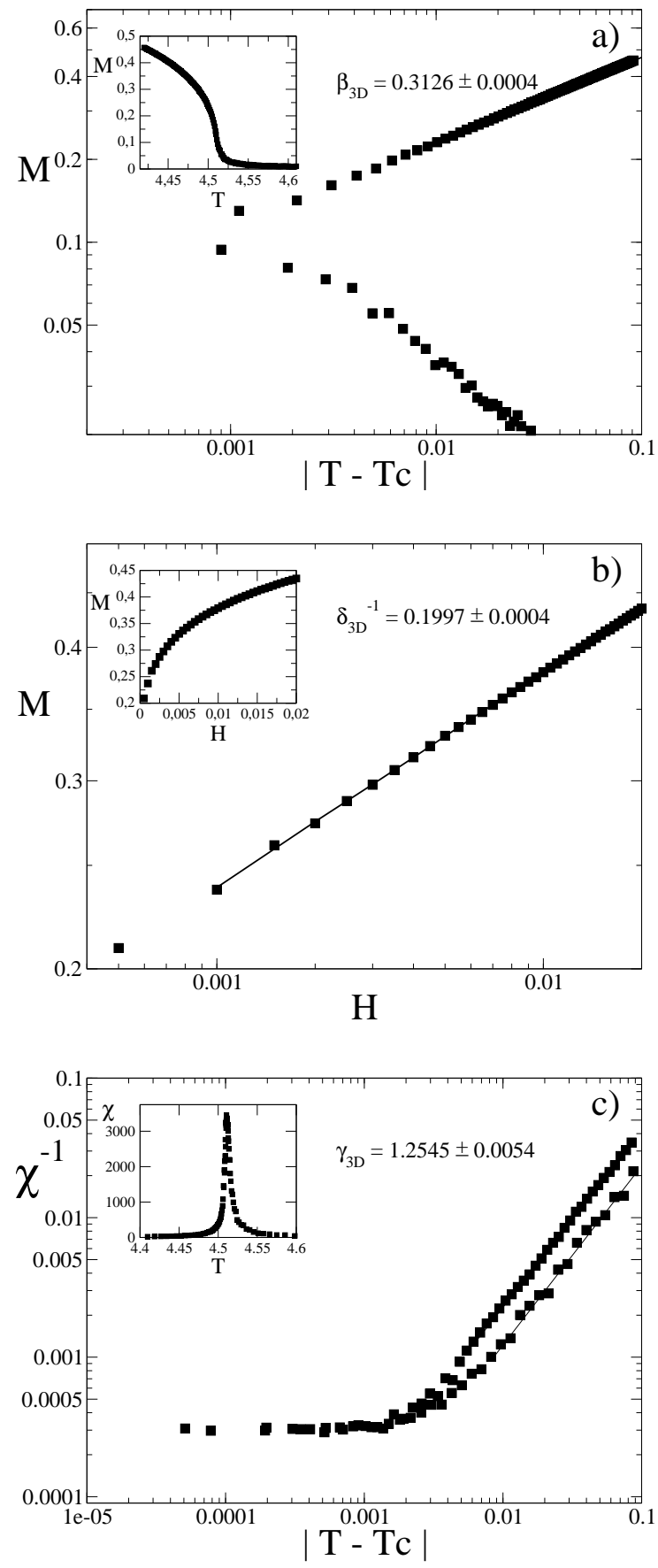

FIG. 3: Three-dimensional critical exponents $\gamma_{3 D}$ (a), $\beta_{3 D}$ (b) and $\delta_{3 D}^{-1}$ (c) obtained by fits of Monte Carlo data of spontaneous magnetization $M_{s}$ near $T_{c}=4.51152 \pm 0.00012$, critical isotherm and isothermal susceptibility $\chi$, respectively, in a system of $L^{3}=115 \times 115 \times 115$ spins with periodic boundary conditions. Insets show the row data. 